\title{
Commentary: Implementing and Evaluating SBIRT for Alcohol Use at a Level 1 Trauma Center: A Behavioral Medicine Approach
}

\author{
Kelly L. Gilrain*, Alexa M. Hays, Victoria A. Grunberg, Anastasia Bullock, Philip Fizur, Steven E. Ross \\ Division of Behavioral Medicine, Cooper University Hospital, USA
}

Article Info

\section{Article Notes}

Received: December 16, 2019

Accepted: January 8, 2020

\section{*Correspondence:}

Dr. Kelly L. Gilrain, Ph.D., Division of Behavioral Medicine,

Cooper University Hospital, One Cooper Plaza, D222, Camden, New Jersey 08103, USA; Telephone No: 856-342-3129; Email: Gilrain-kelly@cooperhealth.edu.

(c) 2020 Gilrain KL. This article is distributed under the terms of the Creative Commons Attribution 4.0 International License.
Alcohol use or overuse often causes many of the injuries both unintentional and intentional - that bring people to hospital emergency rooms and trauma centers each year. These include injuries from motor vehicle accidents, burns, poisoning, and falls as well as violence against oneself or others. Nearly a third (range 26-62\%) of trauma patients have an elevated blood alcohol concentration (BAC) at the time of trauma admission ${ }^{1}$. There has also been a positive association between alcohol use and repeated admission for traumatic injury with an estimated $41 \%$ of trauma recidivism related to the use of alcohol ${ }^{2}$. To address this, the American College of Surgeons Committee on Trauma (ACS-COT) instituted a mandate that all level 1 trauma centers have a mechanism to (1) screen patients for alcohol misuse and (2) provide a brief intervention to those who screen positive ${ }^{3}$. The Screening, Brief Intervention, and Referral to Treatment (SBIRT) risk reduction model has become the most widely endorsed public health approach to improve the detection of and intervention for alcohol misuse in acute care settings ${ }^{4}$. The goal of SBIRT is to use universal screening ( $\mathrm{S}$ ) to identify those at risk for alcohol and other substance use disorders, administer an appropriate brief intervention (BI), and initiate referral to treatment (RT) ${ }^{5}$. SBIRT is an important and effective injury prevention strategy aimed at reducing risky alcohol use ${ }^{6}$. For this particular research, we opted to utilize this intervention due to its support in the literature as well as the fact that it is able to be delivered in a time limited fashion in an acute care setting. Upon admission to Cooper University Hospital (CUH) for a traumatic injury, medical patients with an elevated blood alcohol concentration (BAC) greater than $0.08 \mathrm{~g} / \mathrm{mL}$ (meaning $0.08 \mathrm{~g}$ of alcohol for every $100 \mathrm{~mL}$ of blood) were referred to the Behavioral Medicine CL service. The Alcohol Use Disorders Identification Test ${ }^{7}$ was selected as the screening tool to assess alcohol use with newly admitted trauma patients. BI included education on the effects of alcohol use and problem-solving strategies to reduce or eliminate use, avoid certain risks (e.g., drinking and driving), and seek help from behavioral health treatment and services in the community. Patients were also assessed for their readiness to change and desire to seek out support to assist with behavioral change. Referrals with information about local behavioral health providers and substance abuse treatment organizations were provided to those willing to receive these supports.

Sixty-five trauma patients (out of 535 who completed the SBIRT program during admission) were able to be reached by phone after discharge and willing to complete the follow-up assessment. Follow- 
up AUDIT scores (which were obtained by completing the AUDIT over the phone) fell in the following risk level groups: $58 \%$ were indicative of low risk, $26 \%$ moderate risk, and $15 \%$ high risk. A little more than $20 \%$ of these trauma patients reported seeking treatment to address their alcohol use following hospitalization at CUH. Nearly $73 \%$ of patients expressed that they made positive modifications to their alcohol consumption patterns, such as reducing amount of alcohol consumed or practicing abstinence, since their discharge. When asked about any hospital readmissions since their hospitalization at CUH for a traumatic event, 12 patients reported that they had been re-hospitalized; however, only 2 of these hospitalizations were related to alcohol misuse. A paired t-test was conducted to examine change in alcohol use Time 1 and Time 2. Results indicated that individuals $(n=65)$ who underwent the current intervention reported significantly lower alcohol use at Time $2(M=7.55, S D=7.37)$, compared to Time $1(M=13.38, S D=9.16), t(64)=5.17, p<.0001$. A bivariate correlation was conducted to examine how BAC at hospital admission was associated with change in AUDIT scores from Time 1 to Time 2. Higher BAC at admission was associated with greater improvement in AUDIT scores (i.e., decline in alcohol use) from Time 1 to Time $2(r=-.27, p=$ .036).

An evaluation of CUH's SBIRT program found significant reductions in trauma patients' AUDIT scores and positive changes in alcohol consumption patterns in the year following hospital admission for traumatic injury. These findings suggest successful implementation of this standard of care and provide support for its continued use, which is in line with previous literature ${ }^{5,6,8}$. Results indicate that about half of patients were willing to accept referrals to address alcohol use and $60 \%$ acknowledged difficulties associated with alcohol consumption. CUH's SBIRT program has a positive clinical impact on attitude and behaviors related to alcohol use. Likewise, patients reported less problematic drinking over time, which highlights the utility of this BMed approach. To improve upon this research and continuation of the SBIRT model and incorporate advances in the field, the following changes are recommended: (1) institute training in CUH's SBIRT program for additional hospital providers, particularly departments that cover weekend shifts, in order to directly see trauma patients only admitted for short durations or conduct screenings by phone, (2) adopt universal screening practices for all trauma admits to address alcohol concerns for patients regardless of their BAC at time of admission, and (3) utilize technology and/or briefer screening measure to ease respondent burden and improve visit efficiency.

\section{References}

1. MacLeod JBA, Hungerford DW. Alcohol-related injury visits: Do we know the true prevalence in US trauma centres? Injury. 2011; 42(9): 922-926.

2. Nunn J, Erdongan M, Green RS. The prevalence of alcohol-related trauma recidivism A systematic review. Injury. 2016; 47(3): 551-558.

3. Committee on Trauma Resources for the optimal care of the injured patient. Chicago IL American College of Surgeons. 2006.

4. Mello MJ, Becker SJ, Bromberg J, et al. Implementing alcohol misuse SBIRT in a national cohort of pediatric trauma centers - a type III hybrid effectiveness-implementation trial. Implementation Science. 2018; 13(1): 35.

5. Barbor TF, McRee BG, Kassebaum PA, et al. Screening, brief Intervention, and referral to treatment (SBIRT): Toward a public health approach to the management of substance abuse. Substance Abuse. 2007; 28 (3): 7-30.

6. Bertholet N, Daeppen JB, Wietlisbach V, et al. Reduction of alcohol consumption by brief alcohol intervention in primary care: Systematic review and meta-analysis. Archives of Internal Medicine. 2005; 165 (9): 986-999.

7. Babor TF, Higgins-Biddle JC, Saunders JB, et al. AUDIT The Alcohol Use Disorders Identification Test: Guidelines for use in primary care 2nd ed Geneva Switzerland World Health Organization. 2001.

8. Field C, Walters S, Marti CN, et al. A multisite randomized control trial of brief intervention to reduce drinking in the trauma care setting: How brief is brief? Annals of Surgery. 2014; 259: 873-880. 